\title{
A case of Marfan syndrome with respiratory insufficiency after combined surgery for both pectus excavatum and acute aortic dissection Type-A
}

\author{
Jun Honda, Norie Sanbe, Tsuyoshi Imaizumi, Rieko Oishi, Yuzo Iseki, Masahiro Murakawa
}

Department of Anesthesiology, Fukushima Medical University School of Medicine, Fukushima, Japan

INTRODUCTION

There are several case reports of simultaneous surgical repair of pectus excavatum and cardiovascular surgery that resulted in improved cardio-respiratory function ${ }^{1}$.

Here, We report a case in which there was difficulty with respiratory management after simultaneous surgical repair of pectus excavatum and aortic dissection

\section{CASE DESCRIPTION}

Patient :42-year-old women, height $156 \mathrm{~cm}$, weight $35 \mathrm{~kg}$

The patient had Marfan syndrome and complicated with severe pectus excavatum and scoliosis.

She was able to lead an everyday life with using home oxygen therapy(HOT)

at $1 \mathrm{~L} / \mathrm{min}$ and adaptive servo ventilation(ASV) at night.

She visited the ER with acute aortic dissection type-A. The Bentall procedure, replacement of the ascending aorta, and surgical repair of pectus excavatum

were performed simultaneously to improve cardiopulmonary function.

Presurgical respiratory function test (93 days before operation ):

VC: $1.15 \mathrm{~L}$, \%VC: 37\%, FEV1.0: 0.91 L, FEV1.0\%: 79\%, PEF:2.7 L/s, \%PEF: $34 \%$

There were no major vital changes during the operation.

INTRAOPERATIVE ANESTHESIA RECORD

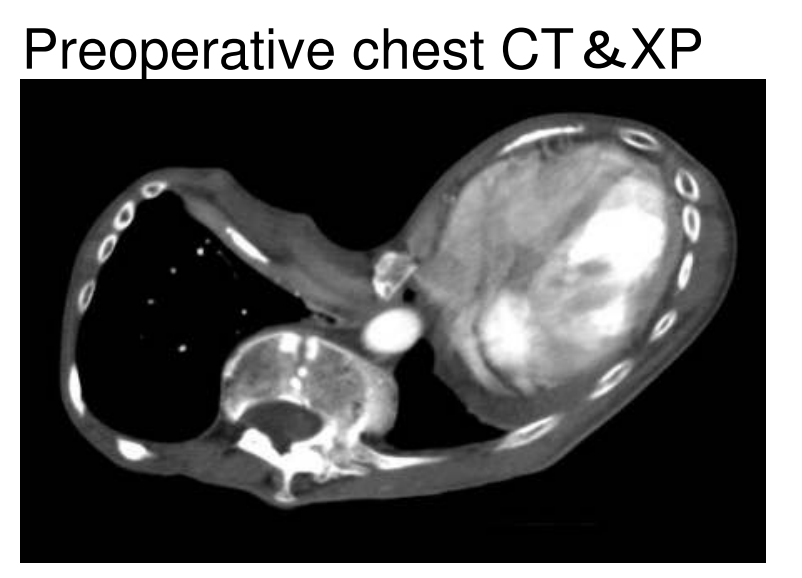

Postoperative chest CT \&

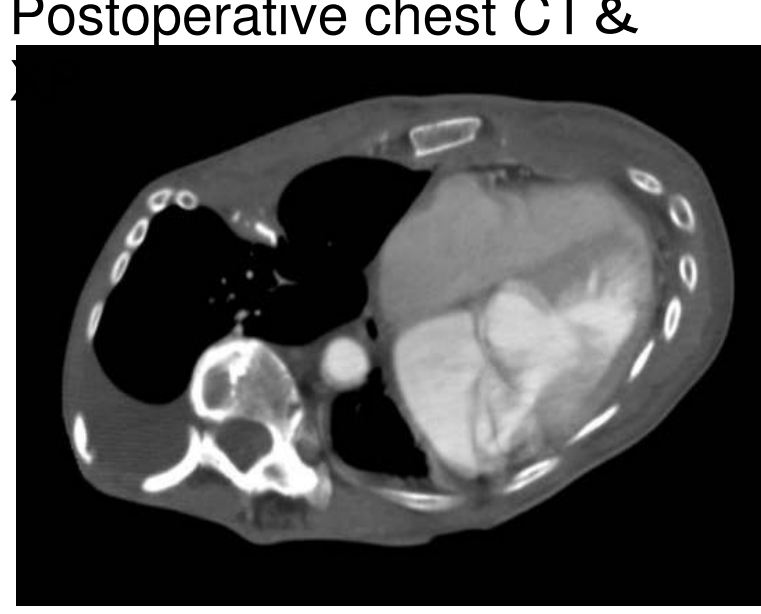

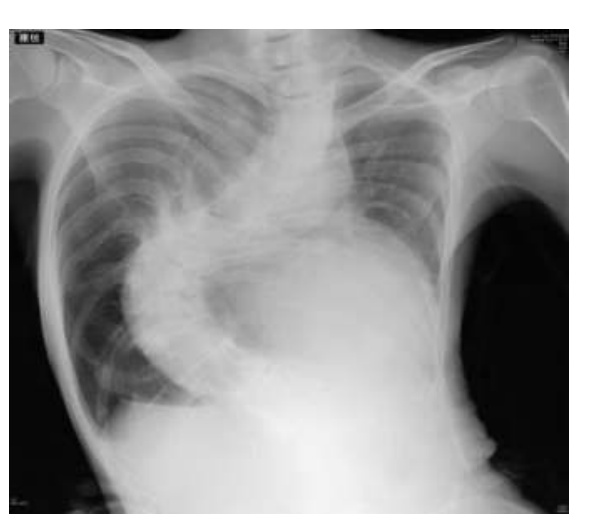

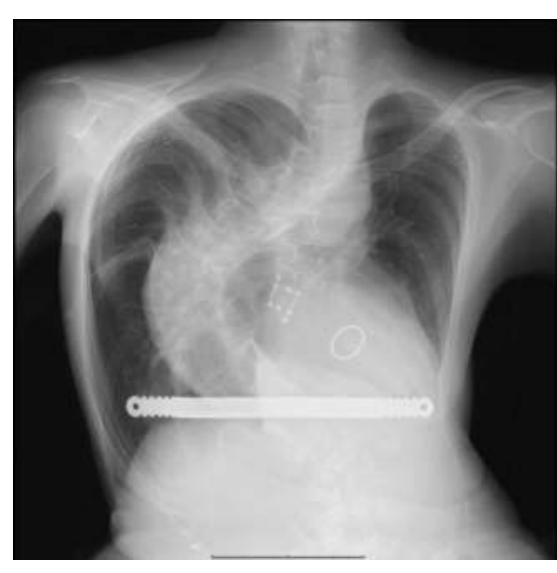

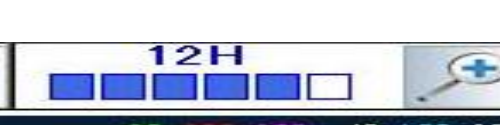
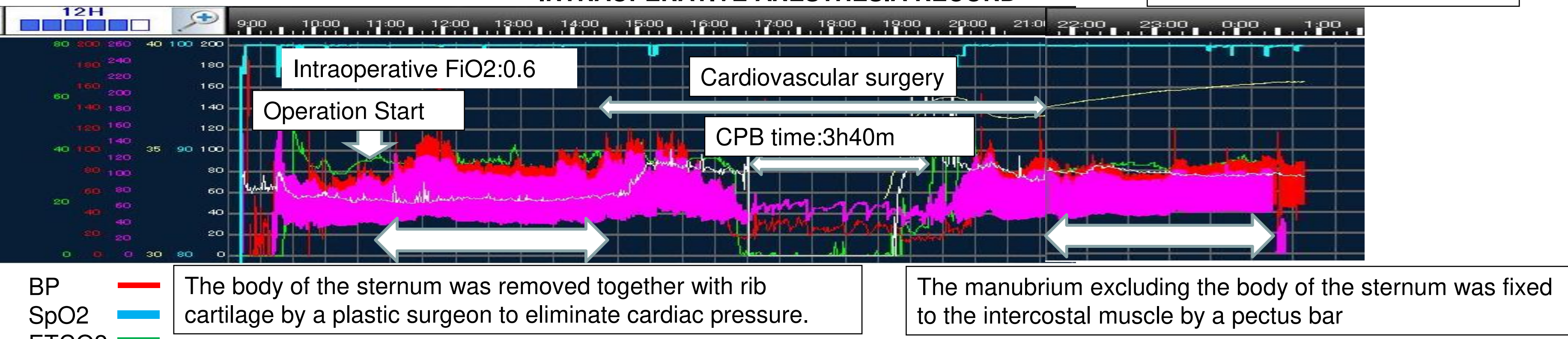

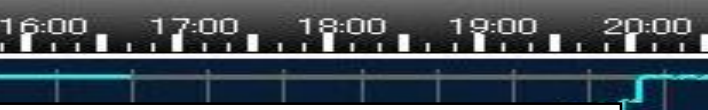

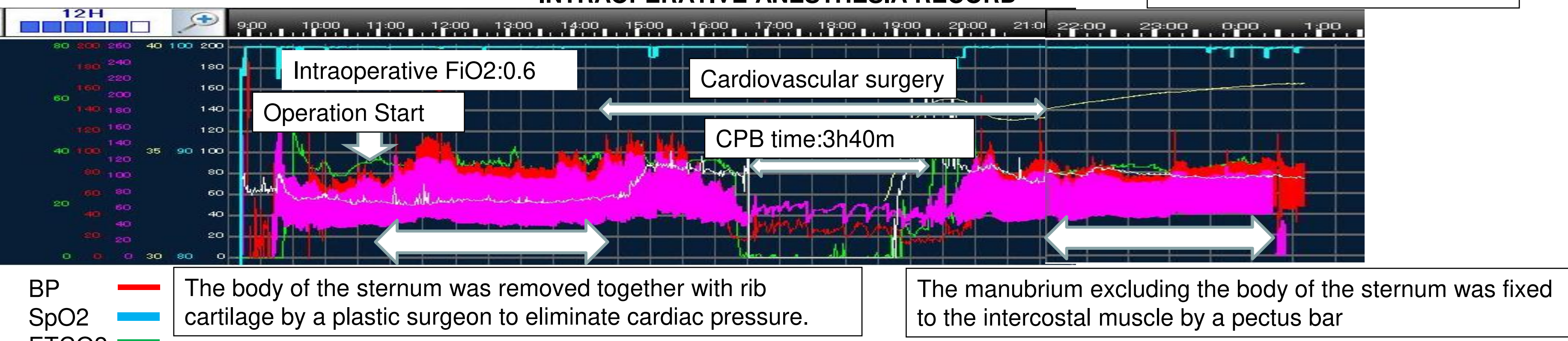

Total Operation Time: 13 h 34 m Anesthesia Time: $15 \mathrm{~h} 52 \mathrm{~m}$

$\mathrm{SpO} 2 \quad$ cartilage by a plastic surgeon to eliminate cardiac pressure.

ETCO2

POSTOPERATIVE COURSE

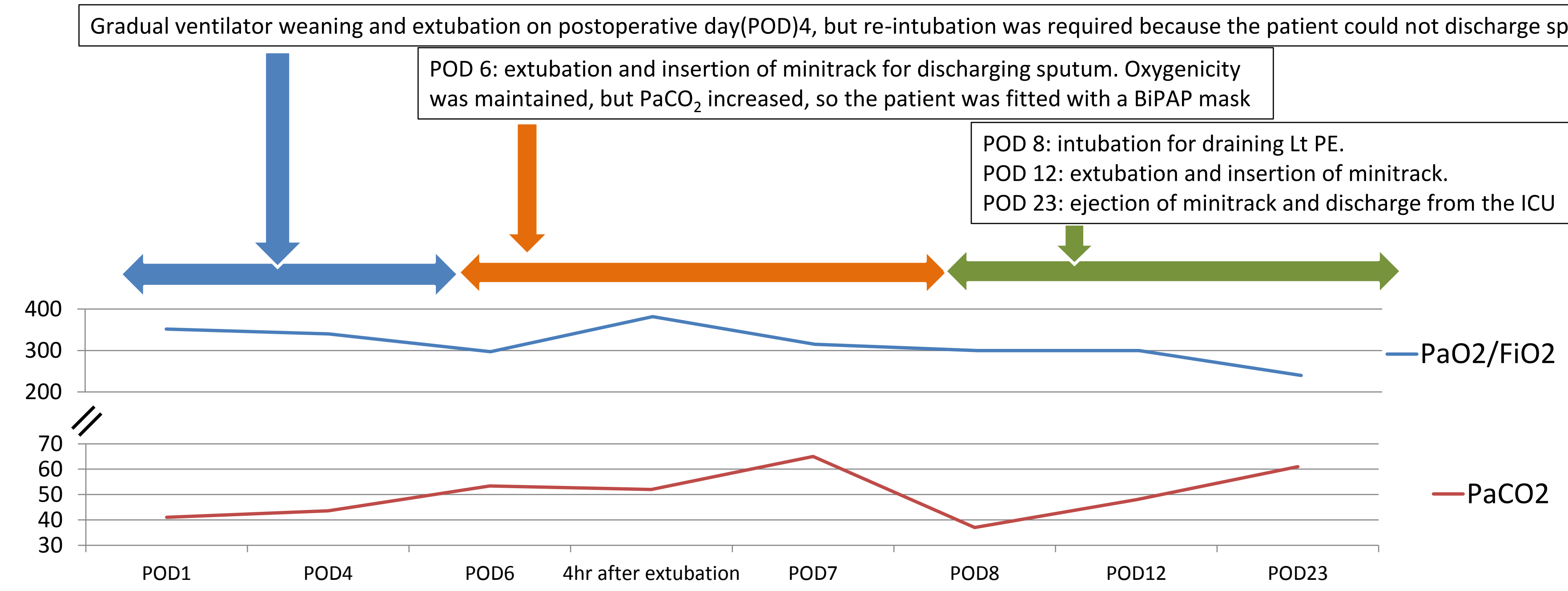

POSTOPERATIVE COURSE

Since cardiovascular status stabilized early after the operation, ventilator support was gradually reduced. The patient was extubated on POD 4.

However, she had difficulty expectorating sputum and had respiratory distress, and tracheal intubation was required again.

On POD 12, the patient was extubated again and managed by mini-track insertion and NPPV. $\mathrm{PaCO}_{2}$ increased to around

70 Torr but P/F was 300 . She was discharged from the ICU on POD 23.

Postsurgical respiratory function test (POD196):

VC: $1.13 \mathrm{~L}$, \%VC: $37.7 \%$, FEV1.0: 0.97 L, FEV1.0\%:

85.8\%, PEF: 3.17 L/s, \%PEF: $40.6 \%$

Ref 1. Combined Repair of Pectus Excavatum and

Cardiopulmonary Bypass Surgery. What Is the Best

Strategy?

Rousse N, at al. Ann Thorac Surg 2013; 96: 1526-7
DISCUSSION

There are several case reports of improved cardio-respiratory function after pectus excavatum repair. In our patient, oxygenation was maintained after surgery, but improvement of respiratory function took time due to restricted chest movement and difficulty with expectoration. Respiratory function was markedly impaired before surgery due to emaciation and deformity, and thus the expected improvement was not achieved by repair of pectus excavatum.

\section{CONCLUSIONS}

This case of simultaneous surgical repair of pectus excavatum and cardiovascular surgery did not result in a marked improvement of respiratory function postoperatively. This was likely due to the emaciated condition of the patient and the limited thoracic mobility after surgery. Therefore, the anticipated improvement of cardio-respiratory function after simultaneous repair of pectus excavatum and cardiac surgery might depend on the patient's condition before surgery. 